\title{
Cost-Aware Cloudlet Placement in Edge Computing Systems
}

\author{
Dixit Bhatta \\ Department of Computer and Information Sciences \\ University of Delaware \\ Newark, DE, USA \\ dixit@udel.edu
}

\begin{abstract}
One of the well-known challenges in Edge Computing is strategic placement of cloudlets. The fundamental goals of this challenge are to minimize the deployment cost and to guarantee minimum latency for the users of edge services. We address this challenge by designing a cost-aware cloudlet placement approach that fully maps user applications to appropriate cloudlets while ensuring their latency requirements. We investigate the effectiveness of our proposed approach by performing extensive experiments based on New York City OpenData. The results show that our approach obtains close to optimal cost solutions with significantly reduced execution time.
\end{abstract}

\section{KEYWORDS}

Cloudlet Placement, Genetic Algorithm, Edge Computing

\section{ACM Reference Format:}

Dixit Bhatta and Lena Mashayekhy. 2019. Cost-Aware Cloudlet Placement in Edge Computing Systems. In The Fourth ACM/IEEE Symposium on Edge Computing (SEC 2019), November 7-9, 2019, Arlington, VA, USA. ACM, New York, NY, USA, 3 pages. https://doi.org/10.1145/3318216.3363369

\section{INTRODUCTION}

Despite improvements in hardware capabilities, it is still a challenge to run computation and data intensive applications on mobile devices as they are restricted by weight, size, battery life, and heat dissipation [6]. Edge computing is a new paradigm that provides a distributed computing solution at the edge of the network, where mobile users consume the computing resources in their vicinity. These resources-rich components placed closer to the users are called Cloudlets [5]. Cloudlets enable task offloading for resource-intensive mobile applications to provide realtime and location-aware services, while reducing traffic to the conventional cloud $[3,7,8]$. Strategically placing these geo-distributed cloudlets to provide low-latency computing services is a major challenge in edge computing. In this paper, we address this challenge by designing a generalized cost-aware cloudlet placement approach that fully maps user applications to appropriate cloudlets and ensures their latency requirements.

Permission to make digital or hard copies of all or part of this work for personal or classroom use is granted without fee provided that copies are not made or distributed for profit or commercial advantage and that copies bear this notice and the full citation on the first page. Copyrights for components of this work owned by others than ACM must be honored. Abstracting with credit is permitted. To copy otherwise, or republish, to post on servers or to redistribute to lists, requires prior specific permission and/or a fee. Request permissions from permissions@acm.org.

SEC 2019, November 7-9, 2019, Arlington, VA, USA

(C) 2019 Association for Computing Machinery.

ACM ISBN 978-1-4503-6733-2/19/11 . \$ \$15.00

https://doi.org/10.1145/3318216.3363369

\author{
Lena Mashayekhy \\ Department of Computer and Information Sciences \\ University of Delaware \\ Newark, DE, USA \\ mlena@udel.edu
}

Multiple studies have investigated the placement of cloudlets with different goals. Wang et al. [9] and Jia et al. [2] have proposed clustering-based approaches to place cloudlets. The focus of the former study is to balance workload and reduce access delay. The latter is based on clustering of users for placing cloudlets in order to minimize response time. Zeng et al. [11] proposed a greedy based algorithm that minimizes the number of placed cloudlets considering latency requirements. A greedy heuristic approach is proposed in [10] to reduce access delay for users served from access points. However, these studies assume all available cloudlets must be placed and do not directly consider placement costs. Moreover, none of these studies designed an approach to assign an individual user or device to a cloudlet.

\section{CLOUDLET PLACEMENT PROBLEM}

As a more comprehensive study, we formulate a generalized cloudlet placement problem with heterogeneous cloudlets and user devices. We model our placement problem in a region represented by a two-dimensional space (grid). We assume only a set of candidate points within the grid are available for placing the cloudlets. The end devices, on the other hand, could be at any point in the twodimensional space. The cloudlets have limited resources and coverage radius, while the devices have computation/storage demands at their specific locations. The cost of placing a cloudlet at a candidate point is given by a cost function. Likewise, a latency function calculates the latency between a device's location and a cloudlet placed at a candidate point. Our goal is to provide edge services to all end devices (full coverage), while minimizing the placement cost for cloudlets and latency for the users.

We formulate the problem, called Optimal Cloudlet Placement (OCP), as a multi-objective integer programming model in a general deployment scenario. The OCP formulation and the constraints suggest a trade-off between cost and latency, and that optimizing one does not always give the best value for the other. This is a bicriteria optimization problem and computationally NP-hard [9]. To tackle mathematical intractability of the problem, we propose a Genetic Algorithm based Cloudlet Placement (GACP), presented in the next section.

\section{GACP APPROACH}

Genetic Algorithm (GA) is an extremely popular method used for solving NP-hard problems. It is a discrete technique that is suitable for combinatorial problems such as grouping, ordering, and assignments. It is especially effective when the problem characteristics are not well understood [4]. In the case of OCP, it is unclear how latency can be smoothly traded-off by favoring placement cost. Moreover, GA has been successfully used in software engineering 


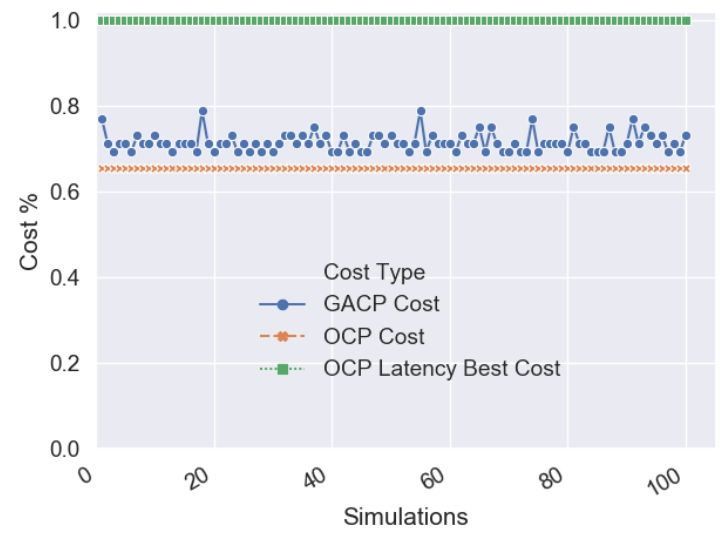

Figure 1: GACP performance: Cost

to generate unit tests with high code coverage [1]. Since covering the end devices to find a complete device to cloudlet mapping is crucial in our problem, GA is the most suitable approach.

GACP initially creates a set of random cloudlet placement and iteratively improves this set of placement. For the devices, GACP maintains an assignment of the devices to the candidate points, where each device is initially assigned to its closest candidate point. This helps in minimizing latency and also in providing a consistent benchmark for coverage calculation for each cloudlet placement.

In each improvement cycle, GACP selects two least-cost cloudlet placements and crossovers them to obtain two offspring, representing the new placements. Then, GACP mutates the two offspring. After that, the selection based on the fitness and coverage values occurs. Since having lower placement cost is our preference, the fitness function is based on the absolute distance of the total placement cost from the optimal placement cost. Since obtaining the optimal cost is NP-hard, GACP uses the linear programming relaxation of OCP to find the estimated optimal cost (tight lower bound) and calculates the fitness.

Once the fitness is calculated, GACP finds the device coverage values. GACP keeps on selecting the cloudlet placements with better costs and coverage values, and discards inferior solutions until it sufficiently improves the candidate solutions. In the end, the least cost cloudlet placement from the improved set of candidate solutions is selected as the final placement solution.

\section{PRELIMINARY RESULTS}

We used NYC WiFi Hotspot Locations and LinkNYC Usage Statistics from NYC OpenData for our experiments. The optimal results from OCP were found using IBM ILOG Concert CPLEX API for Java. The GACP was implemented in the same version of Java, and the experiments for both were run on the same JVM.

We ran each experiment 100 times to capture the distribution of costs, latency, and running time compared to the optimal values. The results based on Central Harlem Data show that the costs obtained by GACP are very close to optimal costs (OCP Cost) as shown in Figure 1. The GACP Cost on average is only $8.8 \%$ more than OCP Cost. Figure 2 shows a very effective cost-latency tradeoff obtained by GACP as the average GACP Latency is $7.8 \%$ less than

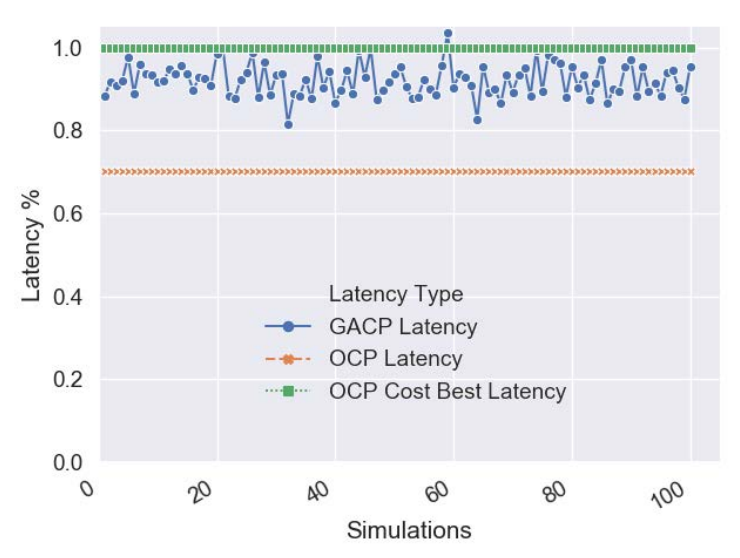

Figure 2: GACP performance: Latency

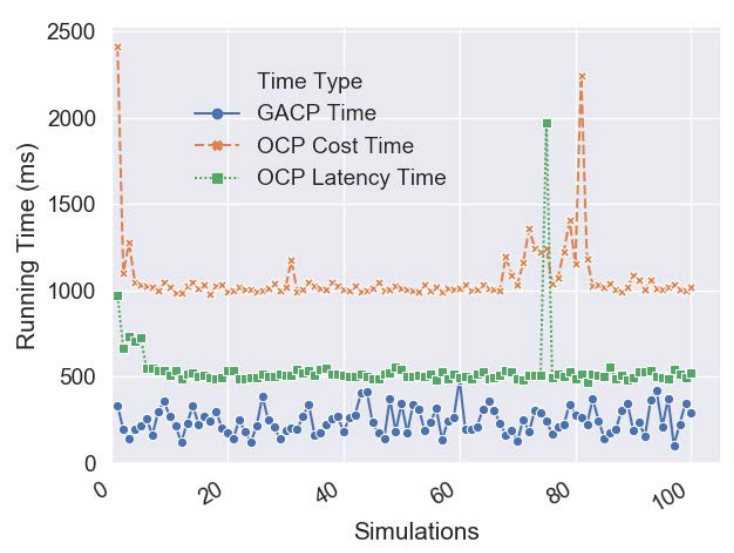

Figure 3: GACP performance: Running Time

the OCP Cost Best Latency. Similarly, Figure 3 shows a significantly lower running time observed by GACP. Note that the extreme spikes in the execution time of OCP are due to NP-hardness of the problem.

The above preliminary results sufficiently demonstrate that GACP is an efficient cost-aware cloudlet placement approach satisfying the desired edge computing requirements. We plan to extend the experiments to include more scenarios to further evaluate the performance of GACP.

\section{CONCLUSION}

Latency suffered by the users can be mitigated by placing resourcerich cloudlets closer to them. However, strategically placing the cloudlets to both reduce costs and ensure all users are covered within their latency requirements is a major challenge in edge computing. We proposed a genetic algorithm-based cloudlet placement approach to address this challenge. Our approach obtains close to optimal solutions and is significantly faster than the optimal implementation. 


\section{ACKNOWLEDGMENTS}

This research was supported in part by NSF grant CNS-1755913.

\section{REFERENCES}

[1] Gordon Fraser and Andrea Arcuri. 2011. EvoSuite: Automatic Test Suite Generation for Object-oriented Software. In Proceedings of the 19th ACM SIGSOFT Symposium and the 13th European Conference on Foundations of Software Engineering (ESEC/FSE '11). ACM, New York, NY, USA, 416-419. https://doi.org/10. $1145 / 2025113.2025179$

[2] M. Jia, J. Cao, and W. Liang. 2017. Optimal Cloudlet Placement and User to Cloudlet Allocation in Wireless Metropolitan Area Networks. IEEE Transactions on Cloud Computing 5, 4 (Oct 2017), 725-737. https://doi.org/10.1109/TCC.2015. 2449834

[3] Weibin Ma, Xuanzhang Liu, and Lena Mashayekhy. 2019. A Strategic Game for Task Offloading among Capacitated UAV-mounted Cloudlets. In Proceedings of the IEEE International Congress on Internet of Things. 1-8.

[4] Melanie Mitchell. 1999. Introduction to Genetic Algorithms. MIT Press, Chapter $1,4,4-20,116-118$.
[5] Mahadev Satyanarayanan. 2017. The Emergence of Edge Computing. Computer (January 2017)

[6] Mahadev Satyanarayanan, Paramvir Bahl, Ramon Caceres, and Nigel Davies. 2009. The Case for VM-Based Cloudlets in Mobile Computing. Pervasive Computing (December 2009).

[7] Nafiseh Sharghivand, Farnaz Derakhshan, and Lena Mashayekhy. 2018. QoSAware Matching of Edge Computing Services to Internet of Things. In Proceedings of the 37th IEEE International Performance Computing and Communications Conference. $1-8$.

[8] Weisong Shi and Schahram Dustdar. 2016. The promise of edge computing. Computer 49, 5 (2016), 78-81.

[9] Shangguang Wang, Yali Zhao, Jinliang Xu, Jie Yuan, and Ching-Hsien Hsu. 2018. Edge server placement in mobile edge computing. F. Parallel and Distrib. Comput. (06 2018). https://doi.org/10.1016/j.jpdc.2018.06.008

[10] Z. Xu, W. Liang, W. Xu, M. Jia, and S. Guo. 2016. Efficient Algorithms for Capacitated Cloudlet Placements. IEEE Transactions on Parallel and Distributed Systems 27, 10 (Oct 2016), 2866-2880. https://doi.org/10.1109/TPDS.2015.2510638

[11] Feng Zeng, Yongzheng Ren, Xiaoheng Deng, and Wenjia Li. 2018. Cost-Effective Edge Server Placement in Wireless Metropolitan Area Networks. Sensors 19 (2018), 32. https://doi.org/10.3390/s19010032 